\title{
Use of telemedicine and e-health in disasters: a systematic review
}

\author{
Simin Salehinejad', Nazanin Jannati ${ }^{2}$, Roghayeh Ershad Sarabi ${ }^{3 *}$, Kambiz Bahaadinbeigy ${ }^{4}$ \\ ${ }^{1}$ Health in Disaster and Emergencies Research Center, Institute for Futures Studies in Health, Kerman University of Medical Sciences, \\ Kerman, Iran \\ ${ }^{2}$ Health Services Management Research Center, Institute for Futures Studies in Health, Kerman University of Medical Sciences, \\ Kerman, Iran \\ ${ }^{3}$ Medical Informatics Research Center, Institute for Futures Studies in Health, Kerman University of Medical Sciences, \\ Kerman, Iran \\ ${ }^{4}$ Modeling in Health Research Center, Institute for Futures Studies in Health, Kerman University of Medical Sciences, Kerman, Iran
}

\begin{abstract}
Received: 13 July 2020
Accepted: 13 September 2020

Published online: 27 September 2020

*Corresponding author: Roghayeh Ershad Sarabi; Assistant Professor, Medical Informatics

Research Center, Institute for Futures

Studies in Health, Kerman University

of Medical Sciences, Kerman, Iran.

Tel: 9133438876

Email: a.ershadsarabi@gmail.com

Competing interests: None.

Funding information: None.

Citation: Salehinejad S, Jannati N, Ershad Sarabi R, Bahaadinbeigy $\mathrm{K}$

Use of telemedicine and e-health

in disasters: a systematic review.

Journal of Emergency Practice

and Trauma 2021; 7(1): 46-62. doi:

10.34172/jept.2020.34
\end{abstract}

\begin{abstract}
Objective: Both natural and man-made disasters need near-term and long-term interventions to reduce morbidity and mortality among the affected population. Recently, telemedicine has been effectively used in both man-made and natural disasters and showed its effectiveness in eliminating the adverse consequences of disasters. Therefore, the purpose of this study was to identify the application of telehealth in different phases of disaster.

Methods: We systematically searched all English papers regarding the use of telemedicine in different phases of disaster indexed in PubMed, Embase, and Web of Science databases until June 2020. A data collection form was designed to extract the required data such as types of systems, telecommunication technologies, and phases of disaster.

Results: In this study, 16 out of 4490 retrieved articles were selected as relevant and reviewed by the authors. Findings indicated that the majority of studies highlighted the use of telemedicine in natural disasters $(n=12)$, and only four discussed it in a man-made disaster like war and terrorist attacks. In 6 studies out of 16, telemedicine was used to treat and assess mental disorders among the affected population. And the internet was the most common telecommunication technology for telemedicine development.

Conclusion: Providing health care during a disaster is necessary, and telemedicine is important for such care. This systematic review helps define the components and application of telemedicine in disaster as a new technology in recent days, but we need to consider it and develop this technology in all phases of disaster management, especially during the response phase.

Keywords: Telemedicine, e-health, Disaster
\end{abstract}

\section{Introduction}

The United Nations has defined disaster as a severe disruption that can change the normal condition and it exceeds the capacity and resources of the affected community (1). Disasters are divided into two basics categories: natural and man-made. Earthquakes, volcanoes, hurricanes, floods, and fires are listed as natural disasters. War, pollution, nuclear explosions, fires, hazardous materials exposures, explosions, and transportation accidents are named as man-made disasters (2). Only in one year, these generated around 40 million homeless and too many deaths in the developed world (3). The cost in this period is about two thousand and a half billion of US dollars (4). These damages sometimes take years to repair, and others can never be repaired.
Since disasters are often a sudden and shocking fact, they require quick and error-free decisions. It is necessary to have pre-determined planning and strategies to respond appropriately, preserve the remaining lives and resources, and reduce pain and suffering. In other words, we need disaster management (5). The International Federation of Red Cross and Red Crescent Societies defines disaster management as to how we "prepare for, respond to, and learn from the effects of major failures" (6).

High costs, inadequate budgets, inappropriate approaches, weakness of alert systems, inappropriate knowledge and motivation, and inadequate awareness of technology are the challenges that disaster management must address them (7). Lack of proper facilities for collecting, processing, and transmitting important 
information is a critical issue that crisis managers face after catastrophic events. (8). If accurate and timely information is made available, unwanted morbidity and mortality may be prevented. Communications are essential requirements to establish contact with the outside world in a disaster management process (9).

It seems that by using IT in disaster management we can detect the risks of disasters and suggest practical solutions in order to save the lives of people and reduce damages (10).

Telemedicine is one of the latest developments in information technology and communications.

Telemedicine, with its unique features, can provide a quick response to essential needs in the event of a disaster (11). For example, in an infectious disease outbreak, remote tracking of care can be utilized quickly and readily through information technology (12)

Army programs, space programs, and various government agencies have gradually developed telemedicine programs, testing them in real, simulated emergencies in the face of civilian disasters (13).

The first application of wireless telemedicine in the disaster was the National Air and Space (NASA) in the 1985 Mexico City earthquake, which destroyed all terrestrial communication infrastructures. The use of advanced communications satellite (ATS-3) voice transmission was the only possible option for international rescue organizations. Also, a space bridge was built during the earthquake in Armenia. The virtual bridge enabled global satellite communications between various countries, including Russia and the United States, in those times (8). Evidence shows that there has been a significant increase in the use of multiple cases of telemedicine successfully in the multinational disasters. With the COVID-19 pandemic of 2020, however, the application of telemedicine and its ability to provide safe, rapid, and high-quality care is apparent (14).

But any action in the use and development of telemedicine must be based on evidence. Its functions and quality of control measures should be evaluated and reported to identify strengths and weaknesses and be integrated into continuous use in critical and standard situations (14).

Therefore, the purpose of this systematic review was to gather information for future research and identify the use of telemedicine in disasters with the following research questions:

1. In which phase of disasters, telemedicine services were used?

2. Which subtype of telemedicine system was used?

3. Which kind of telecommunication technology was employed?

\section{Methods}

This systematic review was undertaken to identify studies focusing on telemedicine and e-health systems in natural and man-made disasters.

\section{Data sources}

We searched PubMed, Web of sciences and, Scopus databases without time limitation for relevant papers in English until May 2020.

\section{Search strategy}

In searching these databases, two groups of key terms were used: (A) key terms denoting disaster concepts including mass disaster, hazard, hurricane, tsunami, disaster, natural disaster, accidents, mass casualty incidents, emergencies, earthquake, avalanche, cyclonic storms, drought, flood, landslide, tidal waves, tornado, and wildfire; (B) key terms denoting telemedicine concepts including telemedicine, telehealth, m-health, mobile health, e-health, telecommunication, and telecare.

To search databases, in advanced search part of each database, we used "OR" to combine terms in groups A and $B$ separately, then, we combined results from two groups using "AND" operator to accumulate all the studies about the telemedicine in disasters (Table 1).

\section{Inclusion and exclusion criteria}

Once the titles and abstracts of the identified articles were obtained, two evaluators independently reviewed and assessed the retrieved publications against the following predefined inclusion and exclusion criteria. Special emphasis was given to articles developing telemedicine or e-health systems in different phases of disaster. Systematic reviews, letter to editor, non-English papers, and studies that did not use telemedicine system in a disaster were excluded from the study.

\section{Data selection, extraction, and analysis}

After searching databases, two reviewers independently selected the articles first by title and abstract and then by full-text review. Then, according to the predefined inclusion and exclusion criteria, relevant articles were selected. Disagreements were discussed between reviewers and in case of any disagreement, a third person was negotiated. Finally, two reviewers extracted relevant information for each article, and then analyzed data using descriptive statistics.

\section{Results}

\section{Summary of the selected literature}

A total of 6300 articles were originally retrieved from the search strategies. A total of 1810 duplicates were removed, leaving 4490 articles to screen. Of these, 16 met our inclusion criteria and were finally analyzed. Publication dates ranged from 2005 to 2018, with the majority of literature published from 2006 to 2020. Important findings were grouped into three main categories: type of telemedicine system, type of telecommunication technology, and the phase of a disaster in which telemedicine was used (Figure 1). 
Table 1. Search strategies in the three databases

"Search $((((()(((($ telemedicine[Title/Abstract]) OR telehealth[Title/Abstract]) OR mhealth[Title/Abstract]) OR "'"mobile health"'[Title/Abstract]) OR m-health[Title/Abstract]) OR e-health[Title/Abstract]) OR telecommunication[Title/Abstract]) OR ehealth [Title/Abstract]) OR telecare[Title/

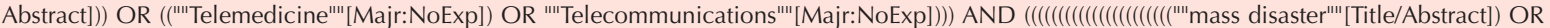
hazard[Title/Abstract]) OR hurricane[Title/Abstract]) OR tsunami[Title/Abstract]) OR disaster[Title/Abstract]) OR "'"natural disaster"'[Title/Abstract])

PubMed OR accidents[Title/Abstract]) OR "'"mass casualty incidents"'"[Title/Abstract]) OR emergencies[Title/Abstract]) OR earthquake[Title/Abstract]) OR avalanche[Title/Abstract]) OR "'"cyclonic storms"'"[Title/Abstract]) OR hurricane[Title/Abstract]) OR drought[Title/Abstract]) OR flood[Title/Abstract]) OR landslide[Title/Abstract]) OR "'tidal waves"'"[Title/Abstract]) OR tsunami[Title/Abstract]) OR tornado[Title/Abstract]) OR wildfire[Title/Abstract])) OR (((()(("'"Disasters"'"[Majr:NoExp])) OR "'"Natural Disasters"'" [Majr]) OR) OR "'"Accidents"'"[Majr:NoExp]) OR "'"Disaster Medicine"'" [Majr:NoExp]) OR "'"Mass Casualty Incidents"'"[Majr:NoExp]) OR "'"Emergencies"'"[Majr:NoExp]))"

\#1 hazard:ti,ab,kw OR tsunami:ti,ab,kw OR disaster:ti,ab,kw OR 'natural disaster':ti,ab,kw OR accident:ti,ab,kw OR 'mass disaster':ti,ab,kw OR emergency:ti,ab,kw OR earthquake:ti,ab, kw OR avalanche:ti,ab,kw OR hurricane:ti,ab,kw OR 'cyclonic storms':ti,ab,kw OR drought:ti,ab, kw OR flood:ti,ab,kw OR landslide:ti,ab,kw OR 'tidal waves':ti,ab,kw OR tsunami:ti,ab,kw OR tornado:ti,ab,kw OR wildfire:ti,ab,kw

Embase \#2 telemedicine:ti,ab,kw OR telehealth:ti,ab,kw OR mhealth:ti,ab,kw OR 'mobile health application':ti,ab,kw OR 'm health':ti,ab,kw OR 'e health':ti,ab,kw OR 'telecare medical information system':ti,ab,kw OR telecommunication:ti,ab,kw OR ehealth:ti,ab,kw OR telecare:ti,ab,kw \#3 \#2 AND \#1

\#1 TOPIC: (hazard) OR TOPIC: (tsunami) OR TOPIC: (disaster) OR TOPIC: ("natural disaster") OR TOPIC: (accident) OR TOPIC: ("mass disaster") OR TOPIC: (emergency) OR TOPIC: (earthquake) OR TOPIC: (avalanche) OR TOPIC: (hurricane) OR TOPIC: ("cyclonic storms") OR TOPIC: (drought) OR TOPIC: (flood) OR TOPIC: (landslide) OR TOPIC: ("tidal waves") OR TOPIC: (tsunami) OR TOPIC: (tornado) OR TOPIC: (wildfire)

wOS \#2 TOPIC: (telemedicine) OR TOPIC: (telehealth) OR TOPIC: (mhealth) OR TOPIC: ("mobile health") OR TOPIC: (m-health) OR TOPIC: (e-health) OR TOPIC: (telecommunication) OR TOPIC: (ehealth) OR TOPIC: (telecare) $\# 3 \# 2$ AND \#1

\section{Geographical focus}

Most of the studies were conducted in united states of america (50\%), and after that in asian countries (43\%) (see Table 2 ). Two studies (11\%) discussed telemedicine and e-health in disasters that happened in the continent of Australia (one in Australia and one in New Zealand). Also, one study addressed the telemedicine system in Africa, including three nations (Guinea, Liberia, and Sierra Leone).

\section{Types of outbreaks}

The use of telemedicine in a disaster was another common thread within this theme. The specific type of disaster addressed most frequently in the reviewed studies was hurricane with 6 citations out of the $16(37.5 \%)$. Three articles (19\%) specifically addressed war, two highlighted the development of telemedicine in the tsunami and two addressed earthquakes. Disasters including bushfire, debris flow, and terrorist attack were just addressed in one study (6\%). Overall, most studies highlighted the use of telemedicine in natural disasters $(75 \%)$, and only four discussed it in man-made disasters like war and terrorist attacks.

\section{Type of telemedicine system and telecommunication technology}

A focus on the type of telemedicine system was the most prominent theme identified in the literature. Six (37\%) papers specifically addressed the telemedicine system used for assessment, diagnosis, and treatment of mental disorders, including depression, anxiety, and PTSD in the post-disaster phase. Six of them were related to the telemedicine system for a visit and triage patients with every symptom during and after a disaster. Tele-radiology, tele-ICU, tele-rehabilitation, and tele-monitoring were discussed accordingly. The results of the studies showed

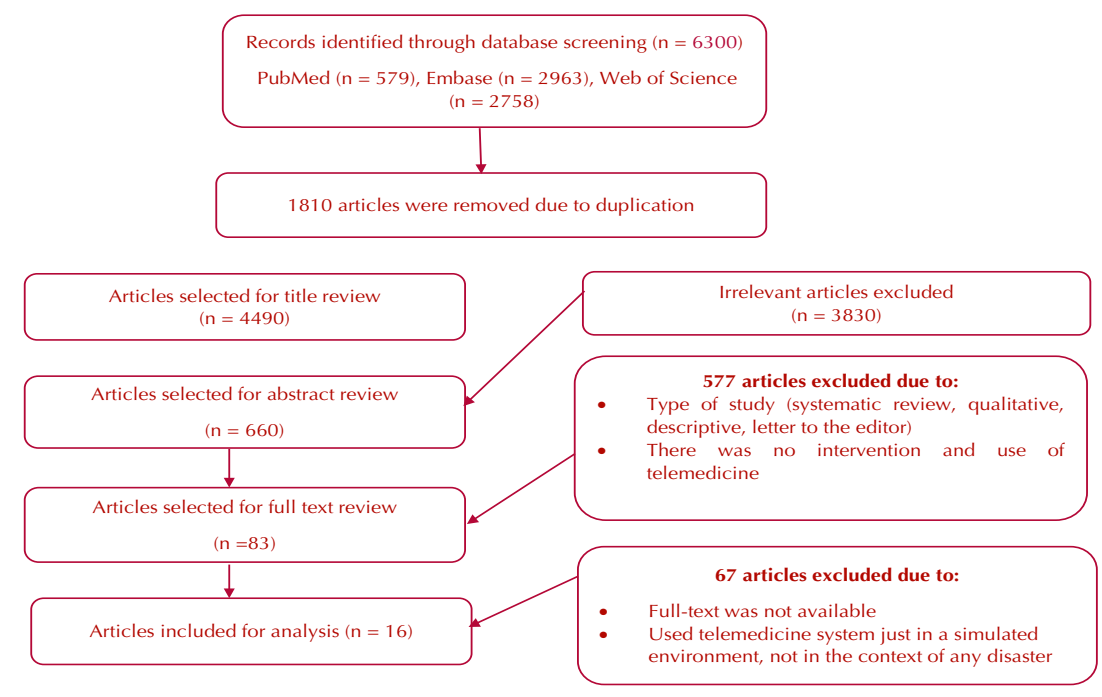

Figure 1. Screening process flowchart. 


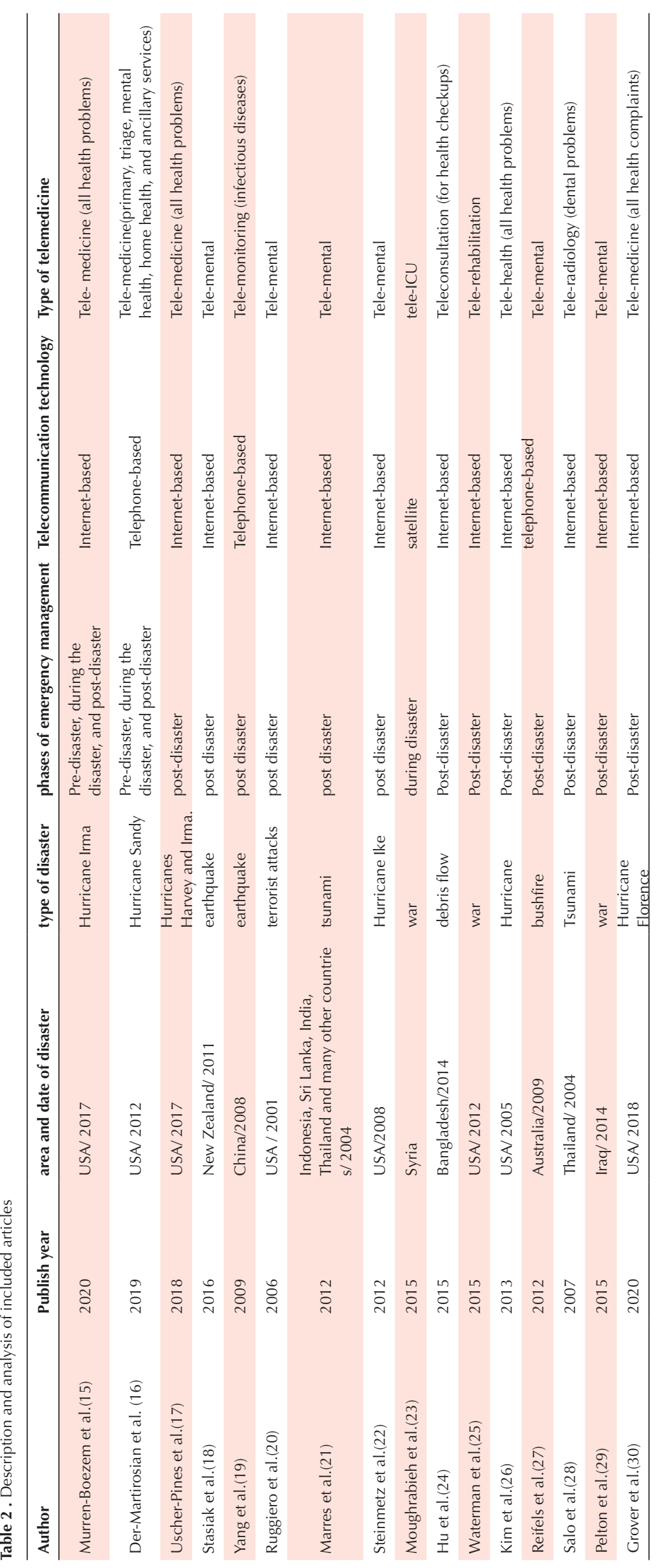


that in 12 studies (75\%), telecommunication technology was internet-based, telephone based technology (call back \& SMS) discussed in two studies and the satellite was the technology used for communication in one study.

\section{Phase of disaster}

Of 16 articles, 13 articles (81\%) focused on the use of telemedicine in post- disaster phase. two articles reported the use of telemedicine services in all three phase of disaster, and in one article, it was used just in during and response phase of disaster.

\section{Discussion}

In disasters, both short-term and long-term interventions are needed to reduce morbidity and mortality among affected populations. Modern Information and communication technologies could be incorporated in disaster preparedness, response and recovery operations and functions (31).

In this systematic review, we investigated the use of telemedicine and e-health systems in four phases of disaster management including reduction, preparedness, response, and recovery. According to our results, in majority of the studies, telemedicine was used in the postdisaster or recovery phase. A short time after the response phase, local governments try to recover residents and get them back to their normal life. For example, experiences of some natural disasters in 2018 show the importance of information systems in coordinating and managing of available resources after response phase (32).

Despite the great potential of telemedicine, it has not been used satisfactorily in all phases of disaster, especially during the disaster and response phases. In most studies, advances in technological communications and telemedicine and its subdomains were in the recovery phase, especially in the field of mental health. The study results show that most of the telemedicine services are related to tele-mental health. It seems that mental disorders are prevalent during or after a disaster. Evidence indicates that survivors of natural disasters may experience several effects on their mental health (33). Common post-traumatic stress responses include psychological distress, physical pain, hyper arousal, behavioral changes, and heightened anxiety (34). After a disaster, many problems can lead to mental disorders. These problems include economic issues, infrastructure damage, house breakdown, and loss of family. These problems have been significant secondary stressors over the subsequent years (35).

On the one hand, early action following a disaster that efficiently reduces such incidents may be of great benefit (33). On the other hand, interventions administered several weeks after the event appear to be related to improved short-term rehabilitation (36). So, tele-mental health can provide early mental health services and help people cope with their psychological problems during or after a disaster. Tele-mental has been used in different disasters, including earthquakes, terrorist attacks, tsunami, and hurricanes.

Telemedicine visits are other kinds of standard telemedicine services. Telemedicine visits can serve millions of patients and support those seeking emergency care through natural disasters (15). During or after a disaster, telemedicine visits may promote treatment that would otherwise be unavailable due to distance, impassable highways, emergency shutdowns, or increased demand for health services (37). Based on our results during two hurricanes, telemedicine visits were used to care for people impacted by this event.

Other telemedicine services have been used in different disasters. These services are tele-ICU, tele-rehabilitation, tele-radiology, tele-monitoring, and tele-health. All these services can be useful in providing diagnosis, treatment, and consultation to survivors of disasters in the early days.

Telemedicine services can be provided on different telecommunication technologies such as the Internet, telephone call, and short message service (SMS). Most telemedicine services are provided using the Internet. An Internet-based approach to telemedicine can offer a cost-effective way of delivering health services and provide a telecommunication technology for long-term care treatment that promotes contact, personalization, and education between patients and physicians (38). Moreover, the Internet can improve healthcare quality and decrease the cost of delivering healthcare (39). The growth of internet-based communication technologies, including email, the worldwide web, and social media telecommunication technologies such as Twitter, Facebook, and YouTube have facilitated telemedicine services (40). Other telecommunication technologies to deliver care are a phone call and SMS. However, these telecommunication technologies have limitations. For example, sending radiology images or medical reports is impossible.

The goal of this study was to discuss the usefulness of telemedicine technology and emphasize that the creation of a disaster preparedness plan that incorporates the available technologies will enhance disaster response efforts.

This study had some limitations due to its explorative nature. The selection of articles may have some reviewer biases. To overcome this limitation, we involved multiple reviewers for the selection and the subsequent analyses. Also, the interpretation of results must be with caution as studies with different methods were included in our investigation.

\section{Conclusion}

In this systematic review we investigated the use of telemedicine in disasters. Based on the obtained results, telemedicine technology was not used in all disaster phases equally. Therefore, efforts should be made to 
encourage the use of telemedicine for effective and quality care in all phases of a disaster, especially in the response and recovery phases. It is now time for policymakers, disaster preparedness teams, technology researchers, and the medical community to create effective disaster management plans to use cost-effective technologies for disaster preparedness, response, and recovery. We believe that the use of information technology such as telemedicine can strengthen disaster managers such as local authorities and policy makers before, during and after disasters.

\section{Authors' Contributions}

SSN, NJ and RES, contributed in searching, reviewing the literature, summarizing the results and writing up the manuscript. $\mathrm{KB}$ also contributed to the design and revision of the manuscript. All authors had a significant contribution to finalizing the manuscript.

\section{Ethics issues}

This study received ethical approval from the vice chancellor for research of the Kerman University of Medical Sciences (IR.KMU.REC.1399.412).

\section{Acknowledgements}

We are grateful to Medical Informatics Research Center of Kerman University of Medical Sciences, Kerman, Iran, for their support in conducting this research.

\section{References}

1. WHO/EHA. Disasters \& Emergencies. Addis Ababa: Panafrican Emergency Training Centre; 2002. Available from: https://apps.who.int/disasters/repo/7656.pdf.

2. Bamgbose OA. An Outline of Natural and Man Made Disaster. Ibadan: Safari Books; 2017.

3. Fang J, Lau CKM, Lu Z, Wu W, Zhu L. Natural disasters, climate change, and their impact on inclusive wealth in G20 countries. Environ Sci Pollut Res 2019; 26(2): 1455-63. doi: 10.1007/s11356-018-3634-2.

4. Grosse SD, Nelson RE, Nyarko KA, Richardson LC, Raskob GE. The economic burden of incident venous thromboembolism in the United States: A review of estimated attributable healthcare costs. Thromb Res 2016; 137: 3-10. doi: 10.1016/j.thromres.2015.11.033.

5. Ghasemi P, Karimian N, editors. A Qualitative Study of Various Aspects of the Application of IoT in Disaster Management. 6th International Conference on Web Research (ICWR); 2020.

6. Abdalla R, Esmail M. Basic concept of disaster management and emergency response. In: WebGIS for Disaster Management and Emergency Response. Cham: Springer; 2019. p. 11-22. doi: 10.1007/978-3-030-03828-1_2.

7. Hosseini SH, Amanat $\mathrm{N}$, Ghanbari $\mathrm{V}$, Nakhaee $\mathrm{M}$, Abbasabadi M, Najafi $M$, et al. Community-based management challenges in disaster risk reduction: a content analysis in Iran. Health in Emergencies and Disasters Quarterly 2017; 2(2): 63-70. doi: 10.18869/nrip.hdq.2.2.63.

8. Ajami S, Lamoochi P. Use of telemedicine in disaster and remote places. J Educ Health Promot 2014; 3: 26. doi: 10.4103/2277-9531.131886.

9. Medford-Davis LN, Kapur GB. Preparing for effective communications during disasters: lessons from a World Health Organization quality improvement project. Int J Emerg Med 2014; 7(1): 15. doi: 10.1186/1865-1380-7-15.

10. Modgil S, Singh RK, Foropon C. Quality management in humanitarian operations and disaster relief management: a review and future research directions. Ann Oper Res 2020: 1-54. doi: 10.1007/s10479-020-03695-5.

11. Doarn CR, Latifi R, Poropatich RK, Sokolovich N, Kosiak D, Hostiuc F, et al. Development and validation of telemedicine for disaster response: the North Atlantic Treaty Organization multinational system. Telemed J E Health 2018; 24(9): 657-68. doi: 10.1089/tmj.2017.0237.

12. Smith AC, Thomas E, Snoswell CL, Haydon H, Mehrotra A, Clemensen J, et al. Telehealth for global emergencies: Implications for coronavirus disease 2019 (COVID-19). J Telemed Telecare 2020; 26(5): 309-13. doi: $10.1177 / 1357633 \times 20916567$.

13. Garshnek V, Burkle FM Jr. Applications of telemedicine and telecommunications to disaster medicine: historical and future perspectives. J Am Med Inform Assoc 1999; 6(1): 2637. doi: 10.1136/jamia.1999.0060026.

14. Latifi R, Doarn CR. Perspective on COVID-19: finally, telemedicine at center stage. Telemed J E Health 2020; 26(9): 1106-9. doi: 10.1089/tmj.2020.0132.

15. Murren-Boezem J, Solo-Josephson P, Zettler-Greeley CM. A pediatric telemedicine response to a natural disaster. Telemed J E Health 2020; 26(6): 720-4. doi: 10.1089/ tmj.2019.0100.

16. Der-Martirosian C, Griffin AR, Chu K, Dobalian A. Telehealth at the US Department of Veterans Affairs after Hurricane Sandy. J Telemed Telecare 2019; 25(5): 310-7. doi: $10.1177 / 1357633 \times 17751005$.

17. Uscher-Pines L, Fischer S, Tong I, Mehrotra A, Malsberger R, Ray K. Virtual first responders: the role of direct-toconsumer telemedicine in caring for people impacted by natural disasters. J Gen Intern Med 2018; 33(8): 1242-4. doi: 10.1007/s11606-018-4440-8.

18. Stasiak K, Merry SN, Frampton C, Moor S. Delivering solid treatments on shaky ground: Feasibility study of an online therapy for child anxiety in the aftermath of a natural disaster. Psychother Res 2018; 28(4): 643-53. doi: 10.1080/10503307.2016.1244617.

19. Yang C, Yang J, Luo X, Gong P. Use of mobile phones in an emergency reporting system for infectious disease surveillance after the Sichuan earthquake in China. Bull World Health Organ 2009; 87(8): 619-23. doi: 10.2471/ blt.08.060905.

20. Ruggiero KJ, Resnick HS, Acierno R, Coffey SF, Carpenter $\mathrm{MJ}$, Ruscio AM, et al. Internet-based intervention for mental health and substance use problems in disasteraffected populations: a pilot feasibility study. Behav Ther 2006; 37(2): 190-205. doi: 10.1016/j.beth.2005.12.001.

21. Marres GM, Leenen LP, van der Slikke JW, Vermetten E. Use of a web portal for support and research after a disaster: opportunities and lessons learned. Interact J Med Res 2012; 1(2): e18. doi: 10.2196/ijmr.1588.

22. Steinmetz SE, Benight CC, Bishop SL, James LE. My Disaster Recovery: a pilot randomized controlled trial of an 
Internet intervention. Anxiety Stress Coping 2012; 25(5): 593-600. doi: 10.1080/10615806.2011.604869.

23. Moughrabieh A, Weinert C. Rapid deployment of international tele-intensive care unit services in wartorn Syria. Ann Am Thorac Soc 2016; 13(2): 165-72. doi: 10.1513/AnnalsATS.201509-589OT.

24. Hu M, Sugimoto M, Hargrave AR, Nohara Y, Moriyama M, Ahmed A, et al. Mobile healthcare system for health checkups and telemedicine in post-disaster situations. Stud Health Technol Inform 2015; 216: 79-83.

25. Waterman BR, Laughlin MD, Belmont PJ Jr, Schoenfeld AJ, Pallis MP. Enhanced casualty care from a global military orthopaedic teleconsultation program. Injury 2014; 45(11): 1736-40. doi: 10.1016/j.injury.2014.03.012.

26. Kim TJ, Arrieta MI, Eastburn SL, Icenogle ML, Slagle M, Nuriddin AH, et al. Post-disaster Gulf Coast recovery using telehealth. Telemed J E Health 2013; 19(3): 200-10. doi: 10.1089/tmj.2012.0100.

27. Reifels L, Bassilios B, Pirkis J. National telemental health responses to a major bushfire disaster. J Telemed Telecare 2012; 18(4): 226-30. doi: 10.1258/jtt.2012.110902.

28. Salo S, Salo H, Liisanantti A, Reponen J. Data transmission in dental identification of mass disaster victims. J Forensic Odontostomatol 2007; 25(1): 17-22.

29. Pelton D, Wangelin B, Tuerk P. Utilizing telehealth to support treatment of acute stress disorder in a theater of war: prolonged exposure via clinical videoconferencing. Telemed J E Health 2015; 21(5): 382-7. doi: 10.1089/ tmj.2014.0111.

30. Grover JM, Smith B, Williams JG, Patel MD, Cabanas JG, Brice JH. Novel use of telemedicine by hurricane evacuation shelters. Prehosp Emerg Care 2020: 1-9. doi: 10.1080/10903127.2020.1723756.

31. Nicogossian AE, Doarn CR. Armenia 1988 earthquake and telemedicine: lessons learned and forgotten. Telemed J E Health 2011; 17(9): 741-5. doi: 10.1089/tmj.2011.0118.
32. Harnesk D. Collective IT artifacts: toward inclusive crisis infrastructures. Journal of Information Technology Theory and Application 2013; 14(4): 27-48.

33. Wang CW, Chan CL, Ho RT. Prevalence and trajectory of psychopathology among child and adolescent survivors of disasters: a systematic review of epidemiological studies across 1987-2011. Soc Psychiatry Psychiatr Epidemiol 2013; 48(11): 1697-720. doi: 10.1007/s00127-013-0731-x.

34. Connor DF, Ford JD, Arnsten AF, Greene CA. An update on posttraumatic stress disorder in children and adolescents. Clin Pediatr (Phila) 2015; 54(6): 517-28. doi: 10.1177/0009922814540793.

35. Lock S, Rubin GJ, Murray V, Rogers MB, Amlôt R, Williams R. Secondary stressors and extreme events and disasters: a systematic review of primary research from 2010-2011. PLoS Curr 2012; 4. doi: 10.1371/currents.dis. a9b76fed1b2dd5c5bfcfc13c87a2f24f.

36. Bryant RA, Harvey AG, Dang ST, Sackville T, Basten C. Treatment of acute stress disorder: a comparison of cognitive-behavioral therapy and supportive counseling. J Consult Clin Psychol 1998; 66(5): 862-6. doi: 10.1037//0022006x.66.5.862.

37. Uscher-Pines L, Fischer S, Chari R. The promise of directto-consumer telehealth for disaster response and recovery. Prehosp Disaster Med 2016; 31(4): 454-6. doi: 10.1017/ s1049023x16000558.

38. Kashem A, Droogan MT, Santamore WP, Wald JW, Marble JF, Cross RC, et al. Web-based Internet telemedicine management of patients with heart failure. Telemed J E Health 2006; 12(4): 439-47. doi: 10.1089/tmj.2006.12.439.

39. Adewale OS. An internet-based telemedicine system in Nigeria. Int J Inf Manage 2004; 24(3): 221-34. doi: 10.1016/j. ijinfomgt.2003.12.014.

40. Tamura T. The internet and personal narratives in the postdisaster anti-nuclear movement. Asia Pac J 2015; 13(7): $1-12$. 